\title{
BlockZoom: Large-Scale Blockchain Testbed
}

\author{
Wazen M. Shbair *, Mathis Steichen *, Jérôme François ${ }^{\dagger}$, Radu State * \\ * University of Luxembourg, SnT, 29, Avenue J.F Kennedy, L-1855 Luxembourg \\ Email:\{wazen.shbair, mathis.steichen, radu.state\}@uni.lu \\ $\dagger$ INRIA Nancy Grand Est, 615 rue du Jardin Botanique, 54600 Villers-les-Nancy, France \\ Email: jerome.francois@inria.fr
}

\begin{abstract}
Future blockchain applications are anticipated to serve millions of users. Thus the evaluation of new blockchain applications have to consider large-scale assessment of the technologies behind the scene. Most of current testing approaches have been done either on simulators or via local small blockchain networks. Hence, the performance in real world conditions is unpredictable. This demonstration introduces BlockZoom, a large-scale blockchain testbed that runs on top of a highly reconfigurable and controllable HPC platform. BlockZoom presents a reproducible environment for experimenting distributed ledgers technologies and smart contract applications. Through different configuration scenarios developers can evaluate the applications performance and the blockchain behavior at a scale comparable to the production environment. The target audience of this demonstration includes researchers and developers in blockchain technology.
\end{abstract}

\section{INTRODUCTION}

Testing and evaluating blockchain application can be conducted using simulation tools, local private networks or via public Testnet networks. The simulation tools are used to prove the viability of an approach [1]. The local private blockchain approach facilitates adapting blockchain configuration parameters. Thus it is possible to reproduce an experiment under different configuration scenarios [2]-[5]. Whereas testing using public testnets mimics conditions that are similar to the production environment. However, the latter two methods have drawbacks; local private blockchains make the testing environment incomparable with real world conditions, while using Testnets obstructs a deep understanding of the employed technology. Accordingly, there is a need for an independent, adaptable and large-scale testing environment.

BlockZoom is a large-scale testbed with adaptable environment built on top of the Grid' 5000 platform [6]. The platform is a large-scale environment for experiment-driven research with large amount of resources scattered over 8 geographic sites in France and Luxembourg. Our aim is to implement a testbed that provides conditions that are close to blockchain production environment, and in parallel has the flexibility of private and local blockchain networks.

\section{DESIGN AND IMPLEMENTATION OVERVIEW}

BlockZoom is designed to gives insight to blockchain developers to validate alternative options. For instance by experimenting with different configurations they can collect performance metrics and compare them with the needed requirements. However, re-configuring a private blockchain network requires significant manual work due to the many components that need to be readjusted. Thus, a tool is required to automate time-consuming and low level details, such that the developers and researchers can focus on the critical questions to answer. In [7] we detailed an orchestration framework for large-scale private blockchain networks. In this work, we extend the orchestration framework with an experiment configuration module as illustrated in Figure 1.

Experiment configuration module. To collect specifications of an experiment from a user, like the number of geographic sites, number of nodes per site, testing duration time, selected blockchain platform and the smart contract which intended to be assessed over the given configurations. Also, the module comes with reconfiguration function, which is used to adapt the configurations of a deployed and running experiment. For instance, the user can modify the number of mining or transaction nodes. In the same context, the workloads reconfiguration function is used to adapt the workloads generator of a smart contract deployed beforehand. Thus, a user can reconfigure the size of workloads, transaction rates or number of working threads to study the behaviour of the blockchain under varying workloads setting. Fine-tuning the blockchain and workloads generator parameters could help the blockchain developers in selecting the right configurations of blockchain platform to meet the needed requirements.

Blockchain orchestration framework. To facilitate the setup of large-scale experiments environment. The framework comprises the nodes reservation module that sends requests based on the inputs come from the configuration module. A given smart contract is deployed and the corresponding workloads generator is launched to send transactions through a blockchain platform. The workloads generator is used as stress testing for both the deployed smart contract and the blockchain platform. The reporting module keeps all the information about the experiment and generates a report with the transactions throughput and latency.

In this demonstration, we will present the evaluation of two smart contracts of small-bank and customers registry with the corresponding workloads generators. The smallbank models banking application where transactions perform deposit and withdraw operations on a customer account. The customers registry simulates client registration operations. For both smart contacts we develop workloads generators that allow one to measure key performance metrics of the smart contracts and the behavior of the Ethereum blockchain under stress conditions. 


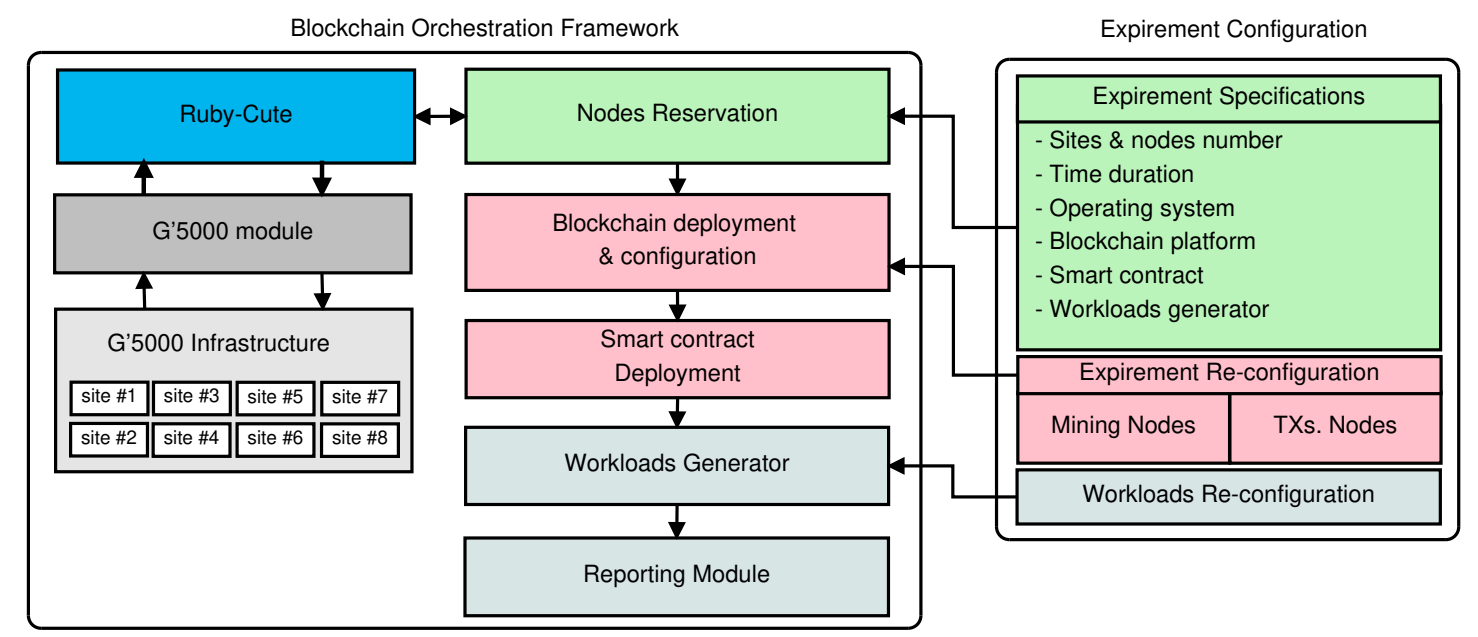

Fig. 1: BlockZoom testbed components: experiment configuration is used to grape an experiment specifications and to reconfigure a running experiment. Blockchain orchestration framework manages the examination environment.

Figure 2 illustrates an example of a private blockchain network infrastructure. It includes five geographic sites with 10 nodes per site, which means in total we have a blockchain network with 50 nodes distributed over a wide geographic area. A user can play with the blockchain configurations, for instance, he/she can dedicate 5 nodes to act like mining nodes and the rest of 45 nodes as transaction nodes, or he/she can reconfigure the workloads generator and accordingly assess the behaviour of the network. The experiment can be repeated with different configurations and then compare the results for evaluating the performance. In summary, we believe that the combination of a well-designed large-scale blockchain testbed with a rich family of workloads is a valuable asset for the blockchain research community.

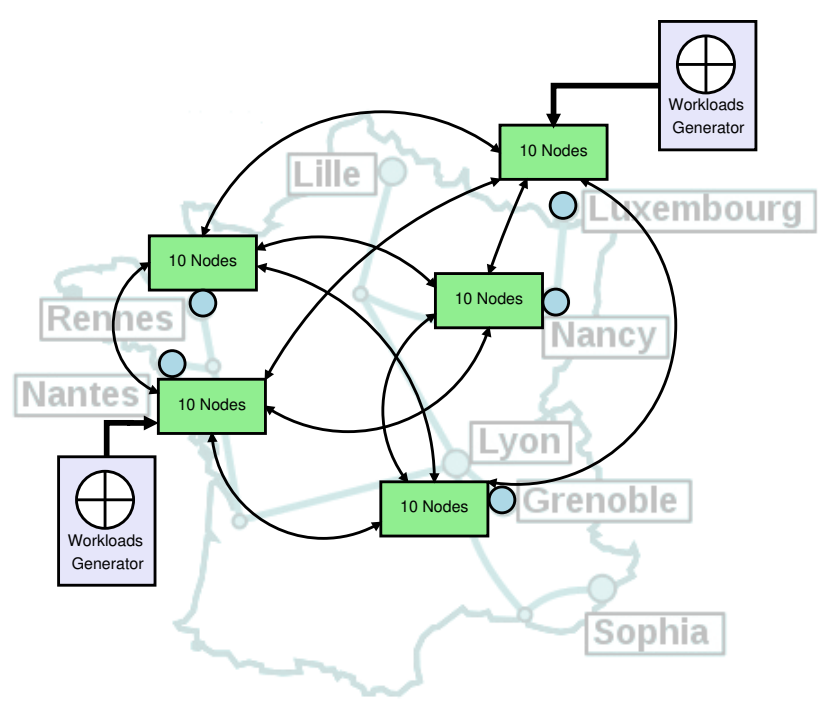

Fig. 2: An example of a blockchain network via BlockZoom.

\section{ACKNOWLEDGEMENTS}

Experiments presented in this paper were carried out using the Grid'5000 testbed, supported by a scientific interest group hosted by Inria and including CNRS, RENATER and several Universities as well as other organizations. The present project is supported by the National Research Fund, Luxembourg under grant agreement number 11785982. Also, this work has received funding from CONCORDIA, the Cybersecurity Competence Network supported by the European Union's Horizon 2020 research and innovation program under grant agreement No 830927.

\section{REFERENCES}

[1] A. Gervais, G. O. Karame, K. Wüst, V. Glykantzis, H. Ritzdorf, and S. Capkun, "On the security and performance of proof of work blockchains," in Proceedings of the 2016 ACM SIGSAC conference on computer and communications security. ACM, 2016, pp. 3-16.

[2] S. Pongnumkul, C. Siripanpornchana, and S. Thajchayapong, "Performance analysis of private blockchain platforms in varying workloads," in 2017 26th International Conference on Computer Communication and Networks (ICCCN), July 2017, pp. 1-6.

[3] T. T. A. Dinh, J. Wang, G. Chen, R. Liu, B. C. Ooi, and K.-L. Tan, "Blockbench: A framework for analyzing private blockchains," in Proceedings of the 2017 ACM International Conference on Management of Data. ACM, 2017, pp. 1085-1100.

[4] P. Thakkar, S. Nathan, and B. Viswanathan, "Performance benchmarking and optimizing hyperledger fabric blockchain platform," in 2018 IEEE 26th International Symposium on Modeling, Analysis, and Simulation of Computer and Telecommunication Systems (MASCOTS). IEEE, 2018, pp. 264-276.

[5] Y. Hao, Y. Li, X. Dong, L. Fang, and P. Chen, "Performance analysis of consensus algorithm in private blockchain," in 2018 IEEE Intelligent Vehicles Symposium (IV). IEEE, 2018, pp. 280-285.

[6] D. Balouek, A. Carpen Amarie, G. Charrier, F. Desprez, E. Jeannot, E. Jeanvoine, A. Lèbre, D. Margery, N. Niclausse, L. Nussbaum, O. Richard, C. Pérez, F. Quesnel, C. Rohr, and L. Sarzyniec, "Adding virtualization capabilities to the Grid'5000 testbed," in Cloud Computing and Services Science, ser. Communications in Computer and Information Science, I. I. Ivanov, M. van Sinderen, F. Leymann, and T. Shan, Eds. Springer International Publishing, 2013, vol. 367, pp. 3-20.

[7] W. Shbair, M. Steichen, J. François et al., "Blockchain orchestration and experimentation framework: A case study of kyc," in The First IEEE/IFIP International Workshop on Managing and Managed by Blockchain (Man2Block) colocated with IEEE/IFIP NOMS 2018, 2018. 